\title{
Non-perturbative renormalization of overlap quark bilinears on domain wall fermion configurations
}

\section{Zhaofeng Liu*i, Ying Chen; Yi-Bo Yang}

Institute of High Energy Physics and Theoretical Physics Center for Science Facilities, Chinese Academy of Sciences, Beijing 100049, China

E-mail: liuzfeihep.ac.cn

\section{Shao-Jing Dong, Michael Glatzmaier, Ming Gong, Keh-Fei Liu}

Department of Physics and Astronomy, University of Kentucky, Lexington, KY 40506

\section{Anyi Li}

Institute for Nuclear Theory, University of Washington, Seattle, WA 98195

Jian-Bo Zhang ${ }^{\S}$

Department of Physics, Zhejiang University, Hangzhou 311027, China

\section{( $\chi$ QCD Collaboration)}

We present renormalization constants of overlap quark bilinear operators on 2+1-flavor domain wall fermion configurations. Both overlap and domain wall fermions have chiral symmetry on the lattice. The scale independent renormalization constant for the local axial vector current is computed using a Ward Identity. The renormalization constants for the scalar, pseudoscalar and vector current are calculated in the RI-MOM scheme. Results in the $\overline{\mathrm{MS}}$ scheme are obtained by using perturbative conversion ratios. The analysis uses in total six ensembles with lattice sizes $24^{3} \times 64$ and $32^{3} \times 64$.

31st International Symposium on Lattice Field Theory LATTICE 2013

July 29 - August 3, 2013

Mainz, Germany

\footnotetext{
* Speaker.

† partially supported by NSFC under Grant 11105153.

\#partially supported by NSFC under Grants 10835002 and 11075167 .

$\S$ partially supported by NSFC under Grant 11275169.
} 


\section{Introduction}

The setup of overlap valence quark on domain wall fermion configurations is successful in lattice calculations of physical quantities such as the strangeness in nucleon [1]. The inversion of overlap fermions can be speeded up by using HYP smearing [2] and deflation with low eigenmodes [3]. The $\chi$ QCD collaboration is determining charm and strange quark masses [4] and other physical quantities using this setup. The renormalization constants of quark bilinear operators needed to match lattice results to those in the continuum $\overline{\mathrm{MS}}$ scheme are calculated in this work.

We use the RI-MOM scheme [5] to calculate renormalization constants for flavor non-singlet scalar, pseudoscalar, vector and axial vector operators $\mathscr{O}=\bar{\psi} \Gamma \psi^{\prime}$, where $\Gamma=I, \gamma_{5}, \gamma_{\mu}, \gamma_{\mu} \gamma_{5}$ respectively. The results are converted to the $\overline{\mathrm{MS}}$ scheme. We have two lattice spacings with various quark masses and give results in the chiral limit of both the valence and light sea quark masses.

\section{Methodology}

The RI-MOM scheme [5] imposes renormalization conditions on amputated Green functions of the relevant operators in the momentum space. The Green functions needed to be computed include the quark propagator, the forward Green function and the vertex function:

$$
\begin{gathered}
S(p)=\sum_{x} e^{-i p x}\langle\psi(x) \bar{\psi}(0)\rangle, \quad G_{\mathscr{O}}(p)=\sum_{x, y} e^{-i p \cdot(x-y)}\langle\psi(x) \mathscr{O}(0) \bar{\psi}(y)\rangle, \\
\Lambda_{\mathscr{O}}(p)=S^{-1}(p) G_{\mathscr{O}}(p) S^{-1}(p) .
\end{gathered}
$$

The renormalization condition is (imposed in the chiral limit)

$$
\left.Z_{q}^{-1} Z_{\mathscr{O}} \frac{1}{12} \operatorname{Tr}\left[\Lambda_{\mathscr{O}}(p) \Lambda_{\mathscr{O}}^{\text {tree }}(p)^{-1}\right]_{p^{2}=\mu^{2}} \equiv Z_{q}^{-1} Z_{\mathscr{O}} \Gamma_{\mathscr{O}}(p)\right|_{p^{2}=\mu^{2}}=1,
$$

where $Z_{q}$ is the quark field renormalization constant with $\psi_{R}=Z_{q}^{1 / 2} \psi$ (the subscript " $\mathrm{R}$ " means after renormalization), $Z_{\mathscr{O}}$ is the renormalization constant for operator $\mathscr{O}$ with $\mathscr{O}_{R}=Z_{\mathscr{O}} \mathscr{O}$ and $\Gamma_{\mathscr{O}}(p)$ is the projected vertex function. In practice, we do calculations at finite quark masses and then extrapolate to the chiral limit. The Green functions in Eq.(2.3) are not gauge invariant, therefore the calculation has to be done in a fixed gauge, usually in the Landau gauge.

We compute the renormalization constant $Z_{A}^{W I}$ of the local axial vector current from a Ward Identity (see Sec. 3.1), which equals to $Z_{A}^{R I}$ in the RI scheme. Then we use

$$
Z_{q}^{R I}=Z_{A}^{W I} \frac{1}{12} \operatorname{Tr}\left[\Lambda_{A}(p) \Lambda_{A}^{\text {tree }}(p)^{-1}\right]_{p^{2}=\mu^{2}} .
$$

to get $Z_{q}^{R I}$, and Eq.(2.3) to compute $Z_{S}, Z_{P}$ and $Z_{V}$ for the scalar, pseudoscalar and vector current. At tree level, $\Lambda_{\mathscr{O}}^{\text {tree }}(p)=\Gamma$ for quark bilinear operators.

The overlap operator [6] is defined as $D_{o v}(\rho)=1+\gamma_{5} \varepsilon\left(\gamma_{5} D_{\mathrm{w}}(\rho)\right)$, where $\varepsilon$ is the matrix sign function and $D_{\mathrm{w}}(\rho)$ is the usual Wilson fermion operator, except with a negative mass parameter $-\rho=1 / 2 \kappa-4$ in which $\kappa_{c}<\kappa<0.25$. We set $\kappa=0.2$ in our calculation that corresponds to $\rho=1.5$. The massive overlap Dirac operator is defined as $D_{m}=\rho D_{o v}(\rho)+m\left(1-\frac{D_{o v}(\rho)}{2}\right)=$ $\rho+\frac{m}{2}+\left(\rho-\frac{m}{2}\right) \gamma_{5} \varepsilon\left(\gamma_{5} D_{w}(\rho)\right)$. More details on our point source overlap fermion propagators can be found in Ref. [3]. With the good chiral properties of overlap fermions, we should and indeed find $Z_{S}=Z_{P}$ and $Z_{V}=Z_{A}$ in our results. 
Table 1: The parameters and statistics of configurations used in this work. The residual masses in lattice units $m_{\text {res }}$ are in the two-flavor chiral limit as given in Ref. [7].

\begin{tabular}{cccccc}
\hline \hline $1 / a(\mathrm{GeV})$ & label & $m_{l} / m_{s}$ & volume & $N_{\text {conf }}$ & $m_{\text {res }}$ \\
\hline $1.73(3)$ & $\mathrm{c} 005$ & $0.005 / 0.04$ & $24^{3} \times 64$ & 92 & $0.003152(43)$ \\
& $\mathrm{c} 01$ & $0.01 / 0.04$ & $24^{3} \times 64$ & 88 & \\
& $\mathrm{c02}$ & $0.02 / 0.04$ & $24^{3} \times 64$ & 138 & \\
\hline $2.28(3)$ & $\mathrm{f} 004$ & $0.004 / 0.03$ & $32^{3} \times 64$ & 50 & $0.0006664(76)$ \\
& $\mathrm{f} 006$ & $0.006 / 0.03$ & $32^{3} \times 64$ & 40 & \\
& $\mathrm{f} 008$ & $0.008 / 0.03$ & $32^{3} \times 64$ & 50 & \\
\hline \hline
\end{tabular}

Table 2: Overlap valence quark masses in lattice units on the $24^{3} \times 64$ and $32^{3} \times 64$ lattices.

\begin{tabular}{ccccccccc}
\hline \hline $24^{3} \times 64$ & 0.00620 & 0.00809 & 0.01020 & 0.01350 & 0.01720 & 0.02430 & 0.03650 & 0.04890 \\
\hline $32^{3} \times 64$ & 0.00460 & 0.00585 & 0.00677 & 0.00885 & 0.01290 & 0.01800 & 0.02400 & 0.03600 \\
\hline \hline
\end{tabular}

\section{Numerical results}

We use configurations generated by the RBC-UKQCD collaboration using $2+1$ flavor domain wall fermions[7, 8]. We employ HYP smearing on the gauge fields and then fix to Landau gauge. The parameters and statistics of configurations are collected in Tab. 1. The overlap valence quark masses are given in Tab. 2. The corresponding pion masses are from about 220 to $600 \mathrm{MeV}$.

We use (anti-)periodic boundary condition in the spacial(time) directions. Thus the momenta are $a p=\left(\frac{\left(2 k_{t}+1\right) \pi}{T}, \frac{2 \pi k_{x}}{L}, \frac{2 \pi k_{y}}{L}, \frac{2 \pi k_{z}}{L}\right)$, where $k_{\mu}=-6,-5, \ldots, 6$ on the $L=24$ lattice and $k_{t}=$ $-5,-1, \ldots, 6, k_{i}=-6,-5, \ldots, 6$ on the $L=32$ lattice. To reduce the effects of Lorentz non-invariant discretization errors, we use the momenta which satisfy the condition $\frac{p^{[4]}}{\left(p^{2}\right)^{2}}<0.32$, where $p^{[4]}=$ $\sum_{\mu} p_{\mu}^{4}$ and $p^{2}=\sum_{\mu} p_{\mu}^{2}$. The statistical errors of our final results are from Jackknife processes.

\subsection{Renormalization of the local axial vector current}

The renormalization constant $Z_{A}$ can be obtained from the axial Ward identity

$$
Z_{A} \partial_{\mu} A_{\mu}=2 Z_{m} m_{q} Z_{P} P
$$

where $A_{\mu}, P$ are the local axial vector current and the pseudoscalar density and $Z_{m}$ is the quark mass renormalization constant with $m_{R}=Z_{m} m_{q}$. Since $Z_{m}=Z_{P}^{-1}$ for overlap fermions, one can find $Z_{A}$ by considering the matrix elements of the both sides of Eq.(3.1) between the vacuum and a pion: $Z_{A} \partial_{\mu}\left\langle 0\left|A_{\mu}\right| \pi\right\rangle=2 m_{q}\langle 0|P| \pi\rangle$. If the pion is at rest, one has $Z_{A}=\frac{2 m_{q}\langle 0|P| \pi\rangle}{m_{\pi}\left\langle 0\left|A_{4}\right| \pi\right\rangle}$, where $A_{4}=\bar{\psi} \gamma_{4} \gamma_{5} \psi$. To obtain the matrix elements, we compute 2-point correlators $G_{P P}(\vec{p}=0, t)=$ $\sum_{\vec{x}}\langle 0|P(x) P(0)| 0\rangle$ and $G_{A_{4} P}(\vec{p}=0, t)=\sum_{\vec{x}}\left\langle 0\left|A_{4}(x) P(0)\right| 0\right\rangle$. Here we follow Ref. [9] closely. When the time $t$ is big, the contribution from the pion dominates in both correlators. Then one has

$$
Z_{A}^{W I}=\lim _{m_{q} \rightarrow 0, t \rightarrow \infty} \frac{2 m_{q} G_{P P}(\vec{p}=0, t)}{m_{\pi} G_{A_{4} P}(\vec{p}=0, t)} .
$$




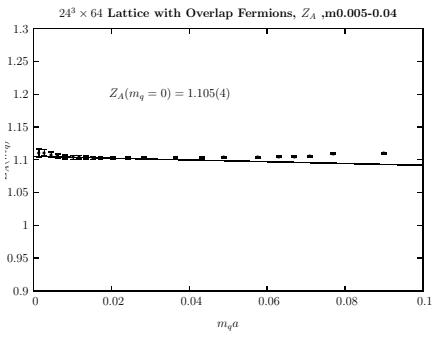

Figure 1: $Z_{A}^{W I}$ against valence quark masses on data ensemble c005.

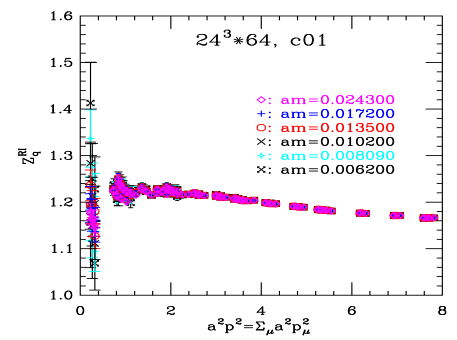

Figure 2: $Z_{q}^{R I}$ as a function of the momentum scale for different valence quark masses on ensemble c01.

Table 3: $\hat{Z}_{A}^{W I}$ on the $24^{3} \times 64$ and $32^{3} \times 64$ lattices.

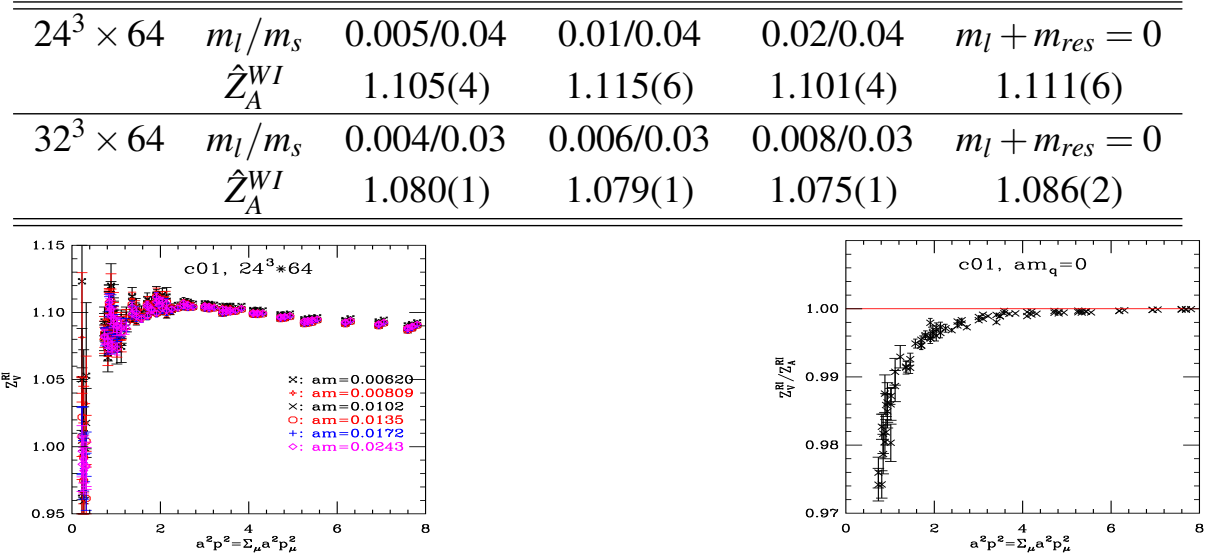

Figure 3: Examples of $Z_{V}^{R I}$ as functions of the momentum scale for ensemble c01.
Figure 4: $Z_{V}^{R I} / Z_{A}^{R I}$ in the valence quark massless limit against the scale for ensembles $\mathrm{c} 01$.

Fig. 1 shows an example of $Z_{A}^{W I}$ obtained from Eq.(3.2) before taking the quark massless limit. For the valence quark massless limit, we fit the data to $Z_{A}^{W I}=\hat{Z}_{A}^{W I}\left(1+b_{A} a m_{q}\right)$. The results of $\hat{Z}_{A}^{W I}$ are given in Tab. 3. In the last column, the results at the light sea quark massless limit are from a linear extrapolation in $m_{l}+m_{\text {res }}$ with $m_{\text {res }}$ given in Tab. 1 .

\subsection{Renormalization constants of the quark field and local vector current}

Fig. 2 shows examples of $Z_{q}^{R I}$ computed from Eq.(2.4) against the scale for various valence quark masses. The quark mass dependence of $Z_{q}^{R I}$ is quite small. In Landau gauge, the anomalous dimension of $Z_{q}$ is zero at 1-loop. This is why $Z_{q}$ is quite flat in Fig. 2.

$Z_{V}^{R I}$ for the local vector current for different valence quark masses on ensemble c01 are shown in Fig. 3. Here in using Eq.(2.3), we have averaged $\mu=1,2,3,4$ for the vector current. The quark mass dependence for $Z_{V}^{R I}$ is small. $Z_{V}^{R I}$ is scale independent at large scale: When $a^{2} p^{2}>\sim 3, Z_{V}^{R I}$ is flat up to discretization errors. Fig. 4 shows the ratio $Z_{V}^{R I} / Z_{A}^{R I}$ for ensemble c01. To go to the chiral limit, we used a linear extrapolation in valence quark masses for $Z_{V}^{R I} / Z_{A}^{R I}$. As we see in Fig. 4, at large momentum scale $Z_{V}^{R I} / Z_{A}^{R I}=1$, i.e., $Z_{V}^{R I}=Z_{A}^{R I}$ is satisfied as expected. The results of $Z_{V}^{R I} / Z_{A}^{R I}$ for other five ensembles are similar.

\subsection{Renormalizations of the scalar and pseudoscalar density}

$Z_{S}^{R I}$ as a function of the scale for different valence quark masses $\left(a m_{q}\right)$ on ensemble c005 are 
Table 4: $Z_{S}^{\overline{\mathrm{MS}}}(2 \mathrm{GeV})$ and $Z_{P, \mathrm{MS}}^{\text {sub }}(2 \mathrm{GeV})$ on the $24^{3} \times 64$ and $32^{3} \times 64$ lattices.

\begin{tabular}{ccccc}
\hline \hline ensemble & $\mathrm{c} 005$ & $\mathrm{c} 01$ & $\mathrm{c} 02$ & $m_{l}+m_{\text {res }}=0$ \\
$Z_{S}^{\overline{\mathrm{MS}}}(2 \mathrm{GeV})$ & $1.1433(54)$ & $1.1397(82)$ & $1.1581(74)$ & $1.1308(87)$ \\
$Z_{P, \overline{\mathrm{MS}}}^{\text {sub }}(2 \mathrm{GeV})$ & $1.164(14)$ & $1.168(22)$ & $1.194(28)$ & $1.141(25)$ \\
\hline ensemble & $\mathrm{f} 004$ & $\mathrm{f} 006$ & $\mathrm{f} 008$ & $m_{l}+m_{\text {res }}=0$ \\
$Z_{S}^{\overline{\mathrm{MS}}}(2 \mathrm{GeV})$ & $1.0607(66)$ & $1.0747(64)$ & $1.077(10)$ & $1.0597(64)$ \\
$Z_{P, \mathrm{MS}}^{S u b}(2 \mathrm{GeV})$ & $1.068(21)$ & $1.093(19)$ & $1.105(24)$ & $1.066(21)$ \\
\hline \hline
\end{tabular}

shown in Fig. 5. Fig. 6 shows $Z_{S}^{R I}$ against $a m_{q}$ at a given scale on ensemble c005. The dependence on $a m_{q}$ is not linear. To go to the chiral limit, we use a 3-parameter function

$$
Z_{S}=\frac{A_{s}}{\left(a m_{q}\right)^{2}}+B_{s}+C_{s}\left(a m_{q}\right)
$$

to fit our data and take $B_{S}$ as the chiral limit value of $Z_{S}$. The double pole term comes from topological zero modes $[10,11]$. In a calculation of $Z_{S}$ in the RI' scheme [12], the curving up of $Z_{S}$ at small $a m_{q}$ is suppressed when the zero modes are subtracted from the quark propagator.

The fits to Eq.(3.3) have small $\chi^{2} /$ dof at all momentum scales. One example of the fits is shown in Fig. 6. $Z_{S}^{R I}$ in the valence chiral limit as a function of the scale for ensemble c005 are shown by the black diamonds in Fig. 7. We use conversion ratios from continuum perturbation theory [13] to 3-loops to convert $Z_{S}^{R I}$ to the $\overline{\mathrm{MS}}$ scheme. The value of $\alpha_{s}^{\overline{\mathrm{MS}}}(\mu)$ in the ratios is obtained by using its perturbative running to 4-loops $[14,15]$. We use $\Lambda_{Q C D}^{\overline{\mathrm{MS}}}=350 \mathrm{MeV}$ for three flavors. $Z_{S}^{\overline{\mathrm{MS}}}$ as a function of the scale $a^{2} p^{2}$ are shown by the red fancy crosses in Fig. 7.

To get $Z_{S}^{\overline{\mathrm{MS}}}(2 \mathrm{GeV})$, we first use the mass anomalous dimension to 4-loops [13] to evolve $Z_{S}^{\overline{\mathrm{MS}}}\left(a^{2} p^{2}\right)$ to $2 \mathrm{GeV}$ (lattice spacings in Tab. 1 are used). The blue crosses in Fig. 7 show $Z_{S}^{\overline{\mathrm{MS}}}(2$ $\left.\mathrm{GeV} ; a^{2} p^{2}\right)$, the running results from the initial scale to $2 \mathrm{GeV} . Z_{S}^{\overline{\mathrm{MS}}}\left(2 \mathrm{GeV} ; a^{2} p^{2}\right)$ would lie on a horizontal line at large $a^{2} p^{2}$ if there were no discretization errors. The solid blue line in Fig. 7 is a linear fit to the blue crosses with $a^{2} p^{2}>5$ to extrapolate away $\mathscr{O}\left(a^{2} p^{2}\right)$ discretization errors. Using the data with $a^{2} p^{2}>4$ to do the extrapolation gives consistent results. $Z_{S}^{\overline{\mathrm{MS}}}(2 \mathrm{GeV})$ on all ensembles are collected in Tab. 4 , where we have used $a^{2} p^{2}>5$ for the extrapolations on the $L=24$ lattices and $a^{2} p^{2}>3$ on the $L=32$ lattices.

From the values on all six ensembles, we do a simultaneous linear extrapolation in the renor-

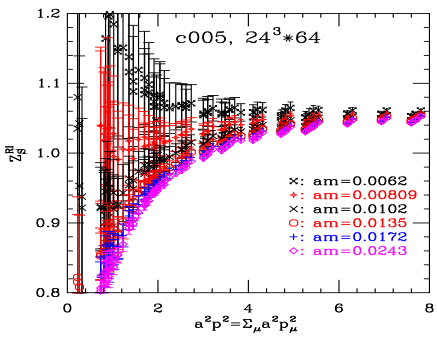

Figure 5: Examples of $Z_{S}^{R I}$ as functions of the momentum scale for ensemble c005.

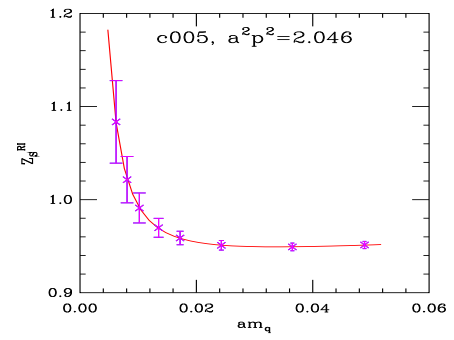

Figure 6: $Z_{S}^{R I}$ against the valence quark mass at a given scale for ensemble c005. 


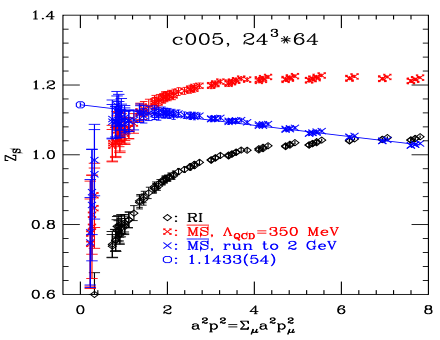

Figure 7: The conversion and running of $Z_{S}$ in the valence quark massless limit on ensemble c005.

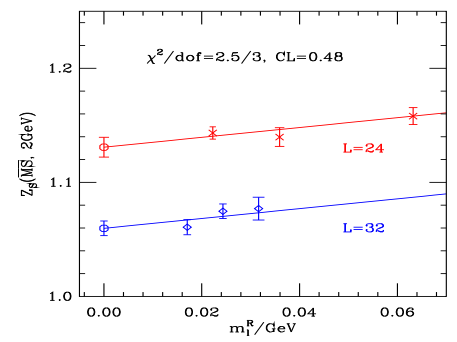

Figure 8: Linear extrapolation of $Z_{S}^{\overline{\mathrm{MS}}}$ to the light sea quark massless limit.

Table 5: Systematic uncertainties of $Z_{S}^{\overline{\mathrm{MS}}}(2 \mathrm{GeV})$ and $Z_{P, \overline{\mathrm{MS}}}^{\text {sub }}(2 \mathrm{GeV})$

\begin{tabular}{|c|c|c|c|c|}
\hline Source & $\begin{array}{c}Z_{S}^{\overline{\mathrm{MS}}}(\mathrm{L}=24) \\
\text { Error }(\%)\end{array}$ & $\begin{array}{c}Z_{S}^{\overline{\mathrm{MS}}}(\mathrm{L}=32) \\
\text { Error }(\%)\end{array}$ & $\begin{array}{c}Z_{P, \overline{\mathrm{MS}}}^{\text {sub }}(\mathrm{L}=24) \\
\text { Error }(\%)\end{array}$ & $\begin{array}{c}Z_{P, \overline{\mathrm{MS}}}^{\text {sub }}(\mathrm{L}=32) \\
\text { Error }(\%)\end{array}$ \\
\hline Truncation (RI to $\overline{\mathrm{MS}})$ & 1.4 & 1.3 & 1.4 & 1.3 \\
\hline Coupling constant & 1.5 & 1.5 & 1.5 & 1.5 \\
\hline Lattice spacing & 0.5 & 0.4 & 0.5 & 0.4 \\
\hline Fit range of $a^{2} p^{2}$ & 0.4 & $<0.1$ & 0.18 & 0.09 \\
\hline Extrapolation in $m_{l}^{R}$ & 0.18 & 1.8 & 0.5 & 3.8 \\
\hline Total & 2.2 & 2.7 & 2.2 & 4.3 \\
\hline
\end{tabular}

malized light sea quark mass to obtain $Z_{S}^{\overline{\mathrm{MS}}}$ in the sea quark massless limit. The fit function is

$$
Z\left(m_{l}^{R}\right)=Z(0)+c \cdot m_{l}^{R}, \quad \text { where } m_{l}^{R}=\left(m_{l}+m_{\text {res }}\right) Z_{m}^{\text {sea }} .
$$

Here $Z_{m}^{\text {sea }}=1.578(2)$ on the $L=24$ lattice and 1.573(2) on the $L=32$ lattice [7]. The slopes in Eq.(3.4) for the coarse and fine lattices are required to be the same. The extrapolation is shown in Fig. 8. The results after extrapolation are given in the last column of Tab. 4. We also did separate linear extrapolations in sea quark masses on the coarse and fine lattices and got consistent results.

The systematic errors of $Z_{S}$ are given in Tab. 5. The $\mathscr{O}\left(\alpha_{s}^{3}\right)$ term in the conversion ratio from the RI-MOM to the $\overline{\mathrm{MS}}$ scheme contributes about $2.2 \%$. The ignored $\mathscr{O}\left(\alpha_{s}^{4}\right)$ term is suppressed by a factor of $\alpha_{s}$. Assuming its coefficient is 3 times as that for the $\alpha_{s}^{3}$ term, we get a $\sim 1.4 \%$ truncation error. Using $\Lambda_{Q C D}^{\overline{\mathrm{MS}}}=400 \mathrm{MeV}$ instead of $350 \mathrm{MeV}$ to evaluate $\alpha_{s}$ changes $Z_{S}^{\overline{\mathrm{MS}}}(2 \mathrm{GeV})$ by $1.5 \%$ on both lattices. The $\mathscr{O}\left(\alpha_{s}^{4}\right)$ term in the perturbative running of $Z_{S}^{\overline{\mathrm{MS}}}$ from an initial scale to $2 \mathrm{GeV}$ contributes less than $0.02 \%$. Thus this truncation error can be ignored. Varying the lattice spacings by one sigma leads to $\sim 0.5 \%$ change in $Z_{S}^{\overline{\mathrm{MS}}}(2 \mathrm{GeV})$. In the extrapolation of $Z_{S}^{\overline{\mathrm{MS}}}(2$ $\mathrm{GeV} ; a^{2} p^{2}$ ) to reduce discretization errors, changing the range of $a^{2} p^{2}$ introduces $0.4 \%$ error on the coarse lattice or $<0.1 \%$ error on the fine lattice. Here we vary $a^{2} p^{2}>5$ to $>4$ on the coarse lattice and $a^{2} p^{2}>3$ to $>2$ on the fine lattice.

Finally we get $Z_{S}^{\overline{\mathrm{MS}}}(2 \mathrm{GeV})=1.131(9)(25)$ on the coarse lattice and 1.060(6)(29) on the fine lattice, where the first error is statistical and the second systematic. The statistical uncertainty is much smaller than the systematic one.

The pseudoscalar renormalization constant $Z_{P}^{R I}$ is shown in Fig. 9 for ensemble c01. The coupling to the Goldstone boson channel [5] leads to the singular behavior in $Z_{P}^{R I}$ at small $a^{2} p^{2}$. To 


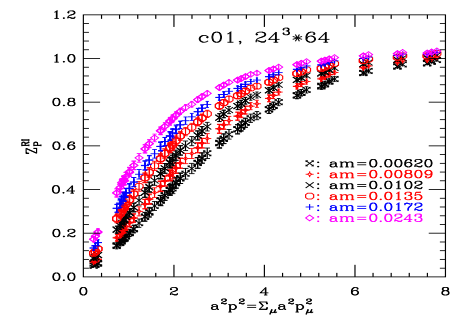

Figure 9: $Z_{P}^{R I}$ against the momentum scale for ensemble c01 at various valence quark masses.

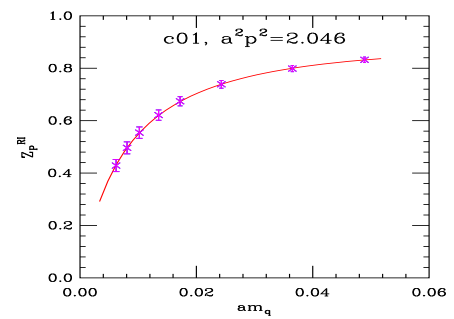

Figure 10: An example of fittings of $Z_{P}^{R I}$ for ensemble c01.

remove this non-perturbative effect, we fit $Z_{P}^{R I}$ at each given $a^{2} p^{2}$ to the 3-parameter ansatz [16]

$$
Z_{P}^{-1}=\frac{A}{a m_{q}}+B+C\left(a m_{q}\right)
$$

and take $Z_{P}^{s u b}=B^{-1}$ as the RI-MOM value in the valence quark chiral limit. Fig. 10 shows one example of the fittings of $Z_{P}^{R I}$ to Eq.(3.5) at a given $a^{2} p^{2}$. All the fittings have small $\chi^{2} /$ dof. $Z_{P}^{s u b}$ is then converted to the $\overline{\mathrm{MS}}$ scheme. Similar to the analysis of $Z_{S}$, we evolve $Z_{P, \overline{\mathrm{MS}}}^{\mathrm{sub}}\left(a^{2} p^{2}\right)$ to $2 \mathrm{GeV}$. Then a linear fit in $a^{2} p^{2}$ to the data at large $a^{2} p^{2}$ is used to reduce $\mathscr{O}\left(a^{2} p^{2}\right)$ discretization errors.

$Z_{P, \mathrm{MS}}^{s u b}(2 \mathrm{GeV})$ on all ensembles are given in Tab. 4 . In the last column, the sea quark massless limit values are obtained from a simultaneous linear extrapolation in the renormalized light sea quark mass with the fit function Eq.(3.4). Comparing the numbers in Tab. 4, we see that $Z_{S}=Z_{P}^{\text {sub }}$ is well satisfied. Similar to the analysis for $Z_{S}$, we give the systematic errors of $Z_{P}^{\text {sub }}$ in Tab. 5 . Unlike $Z_{S}$, the statistical error of $Z_{P, \overline{\mathrm{MS}}}^{s u b}$ is about the same size as the systematic one.

\section{References}

[1] M. Gong et al., Phys. Rev. D 88, 014503 (2013) [arXiv:1304.1194 [hep-ph] ] .

[2] A. Hasenfratz and F. Knechtli, Phys. Rev. D 64, 034504 (2001) [hep-lat/ 0103029 ].

[3] A. Li et al. [xQCD Collaboration], Phys. Rev. D 82, 114501 (2010).

[4] Yi-Bo Yang et al., under preparation.

[5] G. Martinelli, C. Pittori, C. T. Sachrajda, M. Testa and A. Vladikas, Nucl. Phys. B 445 (1995) 81.

[6] H. Neuberger, Phys. Lett. B 417, 141 (1998) [hep-lat/970 07022 ].

[7] Y. Aoki et al. [RBC and UKQCD Collaborations], Phys. Rev. D 83, 074508 (2011).

[8] C. Allton et al. [RBC-UKQCD Collaboration], Phys. Rev. D 78, 114509 (2008).

[9] J. B. Zhang et al., Phys. Rev. D 72, 114509 (2005) [hep-lat/ 0507022 ]

[10] T. Blum et al., Phys. Rev. D 66, 014504 (2002) [hep-lat/0102005] .

[11] Y. Aoki et al., Phys. Rev. D 78, 054510 (2008) [arXiv:0712.1061 [hep-lat] ].

[12] T. A. DeGrand and Z. -f. Liu, Phys. Rev. D 72, 054508 (2005) [hep-lat/ 0507017 ].

[13] K. G. Chetyrkin and A. Retey, Nucl. Phys. B 583 (2000) 3 [arXiv: hep-ph/9910332] .

E. Franco and V. Lubicz, Nucl. Phys. B 531 (1998) 641 [arXiv: hep-ph/9803491] .

[14] A. I. Alekseev, Few Body Syst. 32, 193 (2003) [hep-ph/ 0211339 ].

[15] T. van Ritbergen, J. A. M. Vermaseren and S. A. Larin, Phys. Lett. B 400, 379 (1997).

[16] D. Becirevic et al., JHEP 0408, 022 (2004). [hep-lat/ 0401033 ] 